\title{
Pouchitis after ileal pouch-anal anastomosis for ulcerative colitis occurs with increased frequency in patients with associated primary sclerosing cholangitis
}

\author{
C Penna, R Dozois, W Tremaine, W Sandborn, N LaRusso, C Schleck, D Ilstrup
}

\begin{abstract}
Primary sclerosing cholangitis (PSC), present in $5 \%$ of patients with ulcerative colitis, may be associated with pouchitis after ileal pouch-anal anastomosis. The cumulative frequency of pouchitis in patients with and without PSC who underwent ileal pouch-anal anastomosis for ulcerative colitis was determined. A total of 1097 patients who had an ileal pouch-anal anastomosis for ulcerative colitis, 54 with associated PSC, were studied. Pouchitis was defined by clinical criteria in all patients and by clinical, endoscopic, and histological criteria in $83 \%$ of PSC patients and $85 \%$ of their matched controls. PSC was defined by clinical, radiological, and pathological findings. One or more episodes of pouchitis occurred in $32 \%$ of patients without PSC and $63 \%$ of patients with PSC. The cumulative risk of pouchitis at one, two, five, and 10 years after ileal pouch-anal anastomosis was $15 \cdot 5 \%$, $22 \cdot 5 \%, 36 \%$, and $45.5 \%$ for the patients without PSC and $22 \%, 43 \%, 61 \%$, and $79 \%$ for the patients with PSC. In the PSC group, the risk of pouchitis was not related to the severity of liver disease. In conclusion, the strong correlation between PSC and pouchitis suggest a common link in their pathogenesis.
\end{abstract}

(Gut 1996; 38: 234-239)

Keywords: pouchitis, ulcerative colitis, primary sclerosing cholangitis.

Division of Colon and Rectal Surgery C Penna

R Dozois

Division of

Gastroenterology

W Tremaine

W Sandborn

N LaRusso

Section of Biostatistics C Schleck

D Ilstrup

Mayo Clinic and Mayo Foundation,

Rochester, Minnesota, USA

Correspondence to:

Correspondence to: 200 First Street SW, Rochester, Minnesota 55905, USA

55905, USA.

9 August 1995 discomfort, varies between 7 and $47 \%$ of patients ${ }^{1-4}$ depending on the duration of follow up. Pouchitis usually occurs in patients operated on for $\mathrm{UC}^{5}$ and has been associated with the presence of extraintestinal manifestations of the disease. ${ }^{6}$ In particular, the presence of concomitant primary sclerosing cholangitis (PSC), a chronic cholestatic syndrome of unknown cause characterised by fibrosing obliteration of the bile ducts, seems to be a significant risk factor for the development of pouchitis. ${ }^{7}$

To further explore the association between PSC and pouchitis, the aims of this study were: (a) to determine if PSC represents an independent risk factor for pouchitis; (b) to compare clinical, endoscopic, and pathological findings of pouchitis in a subset of patients without PSC; and (c) to search for correlations between the risk of pouchitis and status of liver disease.

\section{Methods}

\section{Patients}

Between January 1981 and April 1993, 1097 patients underwent ileal J pouch-anal anastomosis for UC at the Mayo Medical Center in Rochester, Minnesota. All pouches were constructed according to a technique previously described, ${ }^{8}$ and patients with indeterminate colitis or with other designs of reservoir were excluded. The following information was retrieved from the medical records of all patients: duration of UC calculated from the date of onset of colonic symptoms to the date of IPAA; extent of UC (rectal, sigmoid, left sided, and pancolonic) and indication for IPAA.

Fifty four of these patients were identified as having associated PSC. The diagnosis of PSC was based on established clinical or biochemical evidence of cholestasis of more than six months' duration and characteristic cholangiographic $^{9}$ or typical hepatic histological findings, or both. ${ }^{10}$ For those patients with PSC, the following information was retrieved from the medical record: (1) Duration of PSC determined by the date of earliest suggested evidence of liver disease. (2) Specific symptoms and signs of liver disease, such as jaundice, fever, cholangitis, encephalopathy, variceal bleeding, ascites, hepatomegaly, splenomegaly, and oesophageal varices. (3) Biochemical testing, including serum alkaline phosphatase, total bilirubin, aspartate aminotransferase, prothrombin time, and serum protein electrophoresis. (4) Cholangiographic appearance of the biliary tree was classified as normal, extrahepatic changes, or intra and extrahepatic changes. ${ }^{11}$ Small duct PSC was 
defined as histological evidence of PSC (pericholangitis) with a normal cholangiogram in a patient with a history of UC. ${ }^{12}$ Histological changes were classified according to the criteria of Ludwig et al ${ }^{13}$ : cholangitis or portal hepatitis (stage I), periportal fibrosis or periportal hepatitis (stage II), septal fibrosis or bridging necrosis, or both (stage III), and biliary cirrhosis (stage IV). Likelihood of patient survival (with regard to PSC) was based on serum bilirubin concentration, histological stage on liver biopsy, age, and the presence of splenomegaly ${ }^{14}$ was also determined for each patient.

\section{Definition of pouchitis and classification of clinical course}

The diagnosis of pouchitis was based on clinical criteria including watery diarrhoea, haematochezia, urgency, abdominal or pelvic discomfort, malaise, and fever. The occurrence of pouchitis in patients with or without PSC was determined by the date of the first episode after IPAA retrieved from a computerised registry. This registry was also used to determine the length of time between IPAA and the first episode of pouchitis, the symptoms of pouchitis, and the number of episodes of pouchitis (less than or equal to two, more than two, or chronic pouchitis).

A more detailed comparison of the clinical, endoscopic, and histological features of all the patients with pouchitis and PSC $(n=34)$ were compared with a group of patients with pouchitis but without PSC $(n=33)$ retrieved from the computerised registry and matched for age, sex, extraintestinal manifestations other than PSC, date of IPAA, and length of follow up after closure of the temporary ileostomy. This more detailed evaluation was performed to assess whether the severity of pouchitis was similar in patients with and without PSC. Clinical evaluation included the length of time between IPAA and the first episode of pouchitis, the symptoms of pouchitis, and the number of episodes of pouchitis as defined above. The following endoscopic findings of inflammation were recorded as present or absent: oedema; granularity; friability; erythema; loss of the vascular pattern; mucus exudate; and mucosal ulceration. ${ }^{15} 16$ The severity of endoscopic inflammation was then classified according to the following definition: mild (discrete oedema of the mucosa); moderate (erythema, mucopurulent exudate, and small ulcerations); or severe (deep ulcerations, diffuse erythema, and extensive mucosa necrosis). Histopathological changes were classified according to the criteria of Shepherd et al. ${ }^{1617}$ The pouchitis disease activity index as described by Sandborn ${ }^{18}$ was also used to quantitate pouchitis disease severity.

Bowel function was obtained from the registry annually updated by a clinical nurse coordinator using telephone contact or questionnaire mailings, or both. Incontinence was described as none, occasional (no interruption of daily activities), or frequent (more than one episode per week with interruption of activities). The use of drugs was defined by the need for more than four days per week of antidiarrhoeal agents or antibiotics to control the frequency of defecation.

\section{Statistical methods}

The association of pouchitis with nominal risk factors was assessed with $\chi^{2}$ tests, the association with ordinal risk factors was assessed with rank sum tests, and the association with continuous variables was assessed with $t$ tests or, when necessary, rank sum tests. The occurrence of pouchitis was estimated as a function of time since surgery using the Kaplan-Meier method. Log rank tests were used to compare the curves with nominal risk factors.

\section{Results}

Cumulative risk of pouchitis after IPAA in UC with or without PSC

Overall, pouchitis occurred at least once in 370 patients $(35 \cdot 7 \%)$ after IPAA. Among the 1043 patients without PSC, pouchitis occurred in 336 patients $(32 \%)$ and in 34 of 54 patients with associated PSC $(63 \%)\left(p<0.001, \chi^{2}\right)$. The estimated risk of pouchitis at one, two, five, and 10 years after IPAA was $15.5 \%$, $22.5 \%, 36 \%$, and $45.5 \%$, respectively, in patients without PSC; and 22\%, 43\%, 61\%, and $79 \%$ in patients with PSC, respectively. Figure 1 shows the occurrence of pouchitis estimated as a function of time. The risk was significantly greater in PSC patients $(p<0.0001, \log$ rank $)$.

\section{Pouchitis disease course after IPAA for UC with} and without PSC

Chronic pouchitis was more frequent in the group of patients with PSC $(60 \% v 15 \%$, $\mathrm{p}<0.001$ ), and acute pouchitis in those patients with less than or equal to two episodes, occurred more often in patients without PSC $(36 \% v 6 \%, \mathrm{p}<0.001)$ (Fig 2).

Clinical, endoscopic, and histological presentation of pouchitis in 34 patients with PSC (group 1) were compared with a group of 33 matched UC patients without PSC (group 2). As Table I shows, both groups were comparable with regard to demographic characteristics.

The mean interval between ileostomy closure and the occurrence of the first episode of pouchitis was 12 months (range one to 96 months) in group 1 and 13 months (range two to 60 months $)$ in group $2(p=0 \cdot 8)$. Diarrhoea was the most common symptom and was present in 94 and $97 \%$ of each group respectively. Abdominal cramping (79\% v 44\%, $\mathrm{p}=0.004)$ and bloody stools (39\% $v 17 \%$, $\mathrm{p}=0.048$ ) were more frequent in group 1 .

Endoscopic examination of the reservoir during or immediately after an episode of pouchitis was performed in 28 patients $(83 \%)$ in group 1 and 28 patients $(85 \%)$ in group 2 . Pouch endoscopy showed similar patterns 


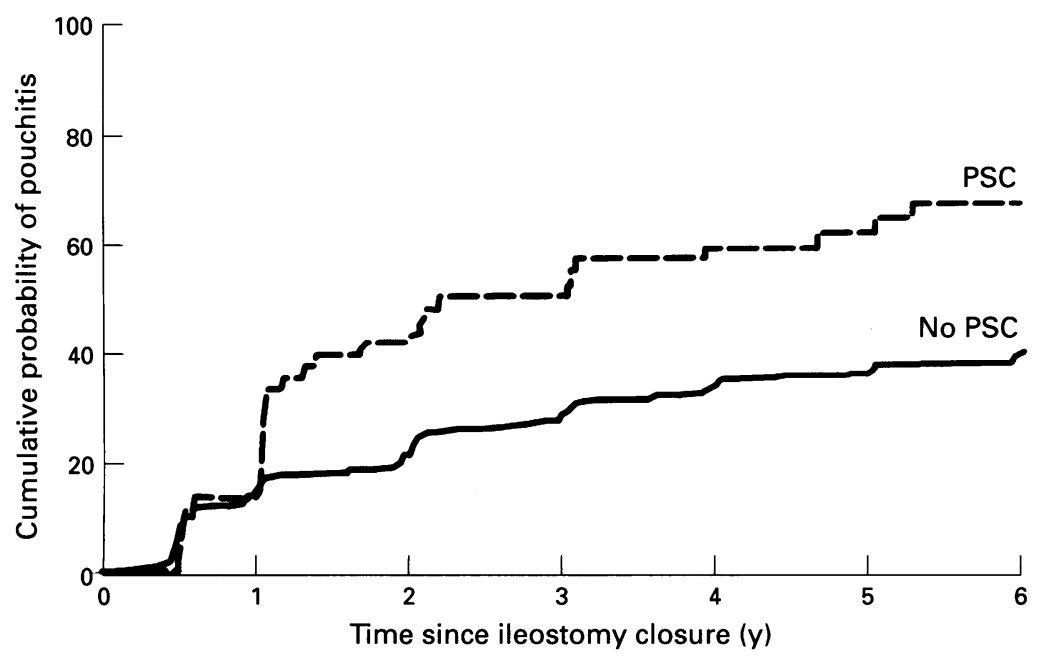

Figure 1: Occurrence of pouchitis estimated as a function of time after closure of temporary ileostomy.

(mild inflammation $(75 \% c 66 \%, \mathrm{p}=0.57)$ and moderate inflammation $(25 \% v 33 \%, \mathrm{p}=0.5)$ in groups 1 and 2, respectively). Specific endoscopic findings of inflammation in groups 1 and 2, respectively included oedema in 24 of 28 patients $(85 \%)$ and 24 of $25(96 \%)$ patients in groups 1 and 2; granularity in 20 of 28 $(71.5 \%)$ and 18 of $25(72 \%)$; friability, 12 of $28(43 \%)$ and eight of $25(32 \%)$; loss of vascular pattern, six of $128(21.5 \%)$ and three of $25(12 \%)$; mucus exudate, 18 of $28(64 \%)$ and 16 of $25(64 \%)$; and ulcerations, 10 of 28 $(36 \%)$ and six of $25(24 \%)$. None of these differences were significant.

The histological score was determined for 28 patients $(83 \%)$ in group 1 and 28 patients $(85 \%)$ in group 2 . Only $50 \%$ (14 of 28 ) of patients with PSC (group 1) and 43\% (12 patients) of patients without PSC (group 2) had a histological score of acute inflammation equal to or superior to 4 and a histological score of chronic inflammation equal to or superior to 4 . The mean overall histological score was $5.6(1.3)$ in group 1 and $5.4(1.2)$ in group $2(p=0.47)$. The pouchitis disease activity index (PDAI) score was greater than or equal to 7 in $93 \%$ of patients in group 1 and $96 \%$ of patients in group 2 , and the mean PDAI score was $8.8(1.6)$ in group 1 and 9.1 (2) in group $2(\mathrm{p}=0 \cdot 8)$.

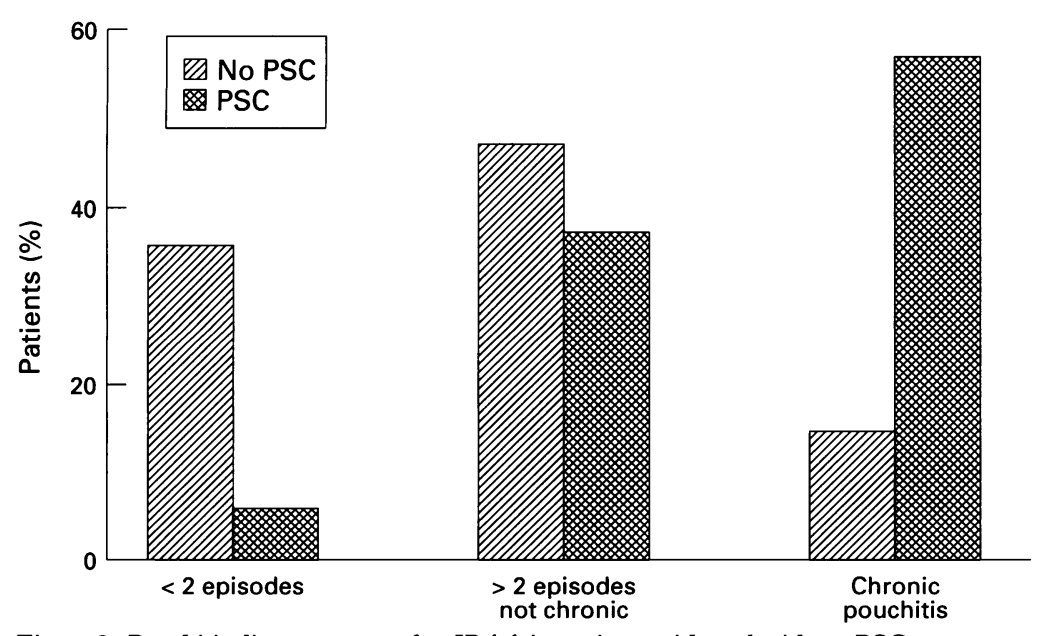

Figure 2: Pouchitis disease course after IPAA in patients with and without PSC.
TABLE I Characteristics of patients with pouchitis I group 1 (PSC) and group 2 (no PSC)

\begin{tabular}{llll}
\hline Characteristics & $\begin{array}{c}\text { Group 1 } \\
(n=34)\end{array}$ & $\begin{array}{l}\text { Group 2 } \\
(n=33)\end{array}$ & $\begin{array}{l}p \\
\text { Value }\end{array}$ \\
\hline Mean (SD) age at CUC (y) & $20(6)$ & $23(6)$ & NS \\
Men:women & $17: 17$ & $17: 16$ & NS \\
Pancolitis (\% of patients) & 97 & 85 & NS \\
Mean (SD) age at IPAA (y) & $31(8)$ & $31(7)$ & NS \\
Number with EIM & 6 & 6 & NS \\
Mean (SD) follow up since & $62(36)$ & $72(33)$ & NS \\
$\quad$ IPAA (months) & & & \\
\hline
\end{tabular}

$\mathrm{CUC}=$ chronic ulcerative colitis, IPAA = ileal pouch-anal anastomosis, EIM=extraintestinal manifestations other than PSC, NS = not significant.

Bowel function after IPAA in pouchitis patients with or without PSC was comparable (Table II); however, more patients in the PSC group had nocturnal stooling, daytime incontinence, and needed drugs.

PSC features and bowel function in PSC patients with and without pouchitis

Among the 54 patients with PSC, 34 developed pouchitis after IPAA (group A, mean follow up 62.5 months, range 12 to 144 ) and 20 patients did not (group B, mean follow up 65 months, range six to 204). There was no difference between the two groups in terms of sex (male to female ratio 17:17 in group $A$ and $11: 9$ in group $B, p=0.8$ ), age at the diagnosis of UC $(20.5(6)$ years $v 25(10)$ years, $\mathrm{p}=0.8)$, presence of pancolonic disease (33 of $34 v 20$ of $20, \mathrm{p}=0 \cdot 8$ ), age at time of IPAA (31 (18) years $v 40$ (9) years, $\mathrm{p}=0.3$ ), or presence of extraintestinal manifestations other than PSC (six of $34 v$ four of $20, \mathrm{p}=0 \cdot 6$ ). The mean (SD) age at the diagnosis of PSC was 30 (8) years (range 14 to 46 ) in patients with pouchitis and 39 (10) years (range 17 to 52 ) in patients without pouchitis $(\mathrm{p}=0.001)$.

As Table II shows, the occurrence of PSC in pouchitis patients did not influence stool frequency or continence. The use of antidiarrhoeal drugs was no greater in patients who experienced pouchitis than in those who did not.

A similar number of patients in each group presented symptoms of liver disease (10 of 34 in group $\mathrm{A} v$ eight of 20 in group $\mathrm{B}, \mathrm{p}=0 \cdot 4$ ) and signs of liver disease (nine of $34 v$ three of 20 for groups $\mathrm{A}$ and $\mathrm{B}$, respectively, $\mathrm{p}=0 \cdot 3$ ). However, splenomegaly $(24 \% v 10 \%)$ and oesophageal varices $(20 \% v 10 \%)$ were more frequent in patients with pouchitis.

Table III shows that mean values of biochemical tests were similar in both groups.

Cholangiograms were performed in 46 of the patients diagnosed with PSC. Of these, 10 $(22 \%)$ had small duct PSC, one $(2 \%)$ had only extrahepatic involvement, and $35(76 \%)$ had evidence of extra and intrahepatic involvement of the biliary tree. Only three of 10 patients with small duct PSC developed pouchitis $(30 \%)$, while 23 of 35 patients with total involvement of the biliary tree subsequently had pouchitis $(66 \%)(p=0.01)$.

Liver biopsy specimens were obtained in 28 patients with pouchitis and 19 patients without pouchitis. Overall, there were 10 stage I, 20 stage II, six stage III, and 11 stage IV. In the 
TABLE II Bowel function of pouchitis patients with or without PSC and of PSC patients without pouchitis

\begin{tabular}{lccc}
\hline & No PSC, & \multicolumn{2}{l}{ PSC } \\
\cline { 3 - 4 } & $\begin{array}{c}\text { pouchitis } \\
(n=33)\end{array}$ & $\begin{array}{l}\text { Pouchitis } \\
(n=34)\end{array}$ & $\begin{array}{c}\text { No pouchitis } \\
(n=20)\end{array}$ \\
\hline Mean (SD) no of stools/24 h (range) & $\begin{array}{c}5 \cdot 5(1 \cdot 9) \\
(3-10)\end{array}$ & $\begin{array}{c}5 \cdot 8(1 \cdot 8) \\
(2-8)\end{array}$ & $\begin{array}{r}5 \cdot 3(1 \cdot 6) \\
(3-10)\end{array}$ \\
No of patients with nocturnal stools (\%) & $26(79)$ & $32(94)$ & $20(100)$ \\
No of patients with normal daytime continence (\%) & $22(67)$ & $24(71)$ & $17(85)$ \\
No of patients with normal night time continence (\%) & $14(42)$ & $12(35)$ & $9(45)$ \\
Drugs & $10(30)$ & $22(65)$ & $11(55)$ \\
\hline
\end{tabular}

group with pouchitis, 15 patients $(54 \%)$ had early hepatic changes (stages I and II) compared with 15 patients $(79 \%)$ in the group without pouchitis $(p=0 \cdot 3)$. Late changes (stages III and IV) were present in 13 patients with pouchitis $(46 \%)$ and in four patients without pouchitis $(21 \%)(p=0 \cdot 3)$.

A risk score could be calculated in 48 patients. The mean (SD) score was 2.79 (1) in the group with pouchitis and $2.73(0.9)$ in the group without pouchitis $(\mathrm{p}=0 \cdot 8, \log \mathrm{rank})$. At follow up, six patients with PSC had died, three each with and without pouchitis, a mean of 54 months after the IPAA and 64 months after the diagnosis of PSC. The estimated survival five years after the diagnosis of PSC was $80 \%$. Cause of death included cholangiocarcinoma in two patients, liver cirrhosis in one patient, and metastatic colon cancer in one patient. Eight patients underwent orthotopic liver transplantation for end stage liver disease and have been described in detail elsewhere. ${ }^{19}$

\section{Discussion}

Our findings show that patients with both UC and PSC have an increased risk of pouchitis after IPAA. Clinical symptoms of inflammation of the pouch occurred in $64 \%$ of 54 patients with PSC and in $32 \%$ of 1043 patients without PSC. Moreover, chronic pouchitis defined by the need for drugs (antibiotics/antiinflammatory drugs) more than 15 days per month to control symptoms was significantly more frequent in the group of patients with PSC. Endoscopic and histological presentation of pouchitis were similar in both groups of patients. We did not find any significant correlation between the severity of the liver disease and the risk of pouchitis in the group of patients with PSC; however, pouchitis was more frequent in patients with diffuse changes on endoscopic retrograde cholangiopancreatography (ERCP) (large duct PSC) and in patients with stage III and IV disease on liver biopsies.

IPAA is a widely accepted procedure for the surgical treatment of ulcerative colitis as $94 \%$

TABLE III Biochemical profile in PSC patients after IPAA

\begin{tabular}{|c|c|c|c|}
\hline \multirow[b]{2}{*}{ Liver tests } & \multicolumn{3}{|c|}{ PSC patients (mean (SD)) } \\
\hline & With pouchitis $(n=34)$ & Without pouchitis $(n=20)$ & p Value \\
\hline Serum bilirubin & $1 \cdot 1(0.5)$ & $1.03(0.7)$ & 0.50 \\
\hline Alkaline phosphatase & $758(585)$ & $575(389)$ & 0.05 \\
\hline Aspartate aminotransferase & $92(64)$ & $69(58)$ & 0.03 \\
\hline Serum albumin & $3 \cdot 8(0 \cdot 6)$ & $3 \cdot 8(0 \cdot 5)$ & 0.87 \\
\hline Prothrombin time & $12 \cdot 2(1 \cdot 2)$ & $12 \cdot 2(1 \cdot 1)$ & $0 \cdot 48$ \\
\hline
\end{tabular}

of patients have a successful outcome over the long term. ${ }^{2}$ As experience with this technique has grown, however, it has become evident that acute pouch inflammation or pouchitis is becoming an important longterm complication. Even with rapid response to treatment, $13 \%$ of all IPAA patients regard pouchitis to be a significant chronic problem with social and professional inconvenience. ${ }^{6}$

The highly variable frequency of pouchitis in published series may be explained by the variety of diagnostic criteria used to define this syndrome and the extent of follow up. In the past, the diagnosis of pouchitis was based solely upon the clinical presentation. ${ }^{256}$ Some authors have advocated the use of endoscopic $^{15}$ or histological criteria ${ }^{1617}$ to confirm a clinical diagnosis of pouchitis. In general, we agree with this approach. ${ }^{1820}$ This viewpoint has not been widely adopted until recently, however, and many of our patients who have been given a clinical diagnosis of pouchitis have not undergone endoscopy with biopsy. In this study, we used clinical criteria to diagnose pouchitis in all 1097 patients. There is some concern that the failure to confirm a clinical diagnosis of pouchitis with endoscopy and histology may lead to an overestimation of the frequency of pouchitis by including patients with irritable bowel syndrome, Crohn's disease, and anastomotic stricture. ${ }^{18}$ This criticism may apply to our patients with a clinical diagnosis of pouchitis who do not have PSC, many of whom did not have a confirmatory endoscopy with biopsies. Most $(83 \%)$ of our patients with PSC and pouchitis, however, had the clinical diagnosis confirmed endoscopically and histologically, making an overestimation of pouchitis in this group unlikely. If the frequency of pouchitis is overestimated in patients without PSC but not in patients with PSC, the net effect would be to decrease the difference in the frequency of pouchitis between the two groups. Thus, the highly significant difference in the frequency of pouchitis between patients with and without PSC in our study may represent a minimal estimate. Based on these data, we believe that the association between PSC and pouchitis is real.

In patients with pouchitis and PSC, and matched controls with pouchitis but without PSC, we found that the use of histological criterion $^{1617}$ alone to diagnose pouchitis was inaccurate in nearly $50 \%$ of patients. This may be explained by a lack of sensitivity and specificity for histological examination. ${ }^{18}$ Pouch inflammation is often patchy and random biopsy specimens may show a great variation of the severity of inflammation within the pouch. Chronic inflammatory changes in the pouch are almost universal, 14 and some degree of acute inflammation is present in $38 \%$ to $64 \%$ of pouches. ${ }^{1621}$ On the other hand, some inflamed pouches with clinical symptoms and endoscopic changes of acute inflammation may fail to show a histological score of inflammation greater than 8 and therefore may not be classified as pouchitis based on histological criteria alone. The $\mathrm{PDAI}^{18}$ may be a better 
indicator of pouchitis. Although this index has not yet been validated, it does seem to be useful in distinguishing patients with and without pouchitis. ${ }^{22-25}$ After a clinical diagnosis of pouchitis has been confirmed with endoscopy and histology, clinical criteria alone may be sufficient for patient treatment as a correlation between the two modes of diagnosis was found in more than $90 \%$ of patients in this study.

The aetiology of pouchitis remains unclear; proposed mechanisms include faecal stasis, perturbation of bacterial flora in the pouch, nutritional deficiencies, ischaemia, and recurrence of UC within the pouch. ${ }^{20}$ Identifying clinical risk factors for pouchitis may help elucidate the aetiology of this disorder. Clinical findings favouring the recurrent UC theory include: the low frequency of pouchitis in patients with familial polyposis and an IPAA ${ }^{5}$; the association of pouchitis with the extraintestinal manifestations of $\mathrm{UC}^{6}$; and the association of perinuclear anticytoplasmic antibodies (pANCA) with UC, PSC, and chronic pouchitis. ${ }^{25-27}$ This study strengthens the recurrent UC theory by showing a strong association between pouchitis and a specific extraintestinal manifestation of UC, PSC. We did not determine the pANCA status of the 1097 patients in this study; however, further study of the inter-relation of pANCA and pouchitis in UC patients with and without PSC certainly seems warranted in further studies. Likewise, studies to determine the HLA genotypes of these patient subgroups should be undertaken.

A changed faecal bile acid pool in patients with PSC could also potentially contribute to the development of pouchitis. In UC patients with an IPAA, there seems to be no difference in the total concentration or total daily output of faecal bile acids between those with and without pouchitis. ${ }^{252829}$ There is some disagreement, however, as to whether bile acid conjugation and dehydroxylation is increased, unchanged or decreased compared with patients without pouchitis. ${ }^{2528} 29$ To date, there are no published reports that bile acids are different in patients with PSC. We did not specifically inquire about the use of ursodeoxycholic acid as a treatment for PSC or the use of cholestyramine as a treatment for pruritus associated with PSC, although it is probable that some of the patients with PSC were taking these drugs. We have previously shown that ursodeoxycholic acid does not improve the disease course of UC in patients with PSC, ${ }^{30}$ and we believed it unlikely to affect the disease course of pouchitis. There was not a correlation between the histological stage of liver disease (stage III or IV) and the development of pouchitis. Thus, treatment with cholestyramine would be unlikely to affect the development or course of pouchitis. The absence of correlation between the risk score of PSC or the histological stage of PSC and the risk of pouchitis lead us to think that the occurrence of pouchitis in PSC patients is dependent upon factors other than the severity of hepatobiliary involvement. Of interest, the risk of pouchitis was greater in patients with diffuse involvement of the biliary tree $(66 \%)$ than in patients with small duct PSC (30\%).

Despite the risk of pouchitis and an increased risk of postoperative complications, IPAA is still our preferred surgical treatment for patients presenting with UC and PSC. After proctocolectomy and Brooke ileostomy, peristomal varices develop in more than $50 \%$ of patients within four years and are often associated with troublesome bleeding. ${ }^{31}$ IPAA can be performed safely in patients with PSC and, thus far, there is no risk of ileoanal anastomotic varices and bleeding. ${ }^{7}$ The high incidence of chronic pouchitis clearly emphasises the need for better treatments; however, this major cause of concern after IPAA seldom requires pouch exclusion or excision.

The authors thank Lorraine Winter for her meticulous record keeping and Jenny Walsh for the preparation of this manuscript. Presented in part at the Annual Meeting of the American Gastroenterology Association, New Orleans, 1994 and published as an abstract in Gastroenterology 1994; 106: A751.

1 Nicholls RJ, Moskowitz FL, Shepherd NA. Restorative proctocolectomy with ileal reservoir. Br F Surg 1985; 72: 576-9.

2 Pemberton JH, Kelly KA, Beart RW Jr, Dozois RR, Wolff BG, Ilstrup DM. Ileal pouch-anal anastomosis for chronic ulcerative colitis. Long-term results. Ann Surg 1987; 206: ulcerative

3 Fleshman JW, Cohen Z, McLeod RS, Stern H, Blair J. The ileal reservoir and ileoanal anastomosis procedure: factors affecting technical and functional outcome. Dis Colon Rectum 1988; 31: 10-6.

4 Svaninger G, Nordgren S, Oresland T, Hulten L. Incidence and characteristics of pouchitis in the Kock continent, ileostomy and the pelvic pouch. Scand $\mathcal{f}$ Gastroenterol 1993; 28: 695-700

5 Dozois RR, Kelly KA, Welling DR, Gordon H, Beart RW $\mathrm{Jr}$, Wolff BG, et al. Ileal pouch-anal anastomosis: comparison of results in familial adenomatous polyposis and
chronic ulcerative colitis. Ann Surg 1989; 210: 268-73.

6 Lohmuller JL, Pemberton JH, Dozois RR, Ilstrup D, van Heerden J. Pouchitis and extraintestinal manifestations of inflammatory bowel disease after ileal pouch-anal anastoinflammatory bowel disease after ileal

7 Kartheuser AH, Dozois RR, Wiesner RH, LaRusso NF, Ilstrup DM, Schleck CD. Complications and risk factors after ileal pouch-anal anastomosis for ulcerative colitis associated with primary sclerosing cholangitis. Ann Surg 1993; 217: 314-20.

8 Ballantyne GH, Pemberton JH, Beart RW Jr, Wolff BG, Dozois RR. Ileal J pouch-anal anastomosis: current technique. Dis Colon Rectum 1985; 28: 197-202.

9 MacCarty RL, LaRusso NF, Wiesner RH, Ludwig J. Primary sclerosing cholangitis: findings on cholangiography and pancreatography. Radiology 1993; 149: 39-44.

10 Ludwig J, Barham SS, LaRusso NF, Elveback LR, Wiesner RH, McCall JT. Morphologic features of chronic hepatitis $\mathrm{RH}, \mathrm{McCall}$ JT. Morphologic features of chronic hepatitis associated with primary sclerosing cholangitis

11 MacCarty RL, LaRusso NF, Wiesner RH, Ludwig J. Primary sclerosing cholangitis: findings on cholangiography and pancreatography. Radiology 1983; 149: 39-44.

12 Wee A, Ludwig J. Pericholangitis in chronic ulcerative colitis: primary sclerosing cholangitis of the small bile ducts? Ann Intern Med 1985; 102: 581-7.

13 Ludwig J, LaRusso NF, Wiesner RH. Primary sclerosing cholangitis. Contemp Issues Surg Pathol 1986; 8: 192-213.

14 Dickson ER, Murtaugh PA, Wiesner RH, Grambsch PM, Fleming TR, Ludwig J, et al. Primary sclerosing cholangitis: refinement and validation of survival models. gitis: refinement and validation of
Gastroenterology 1992; 103: 1893-901.

15 Tytgat GNJ. The role of endoscopy in pouch monitoring and pouchitis: pouchitis workshop. Int $\mathcal{f}$ Colorectal Dis 1989; 4: 205-29.

16 Moskowitz RL, Shepherd NA, Nicholls RJ. An assessment of inflammation in the reservoir after restorative proctocolectomy with ileoanal ileal reservoir. Int $\mathcal{f}$ Colorectal Dis 1986; 1: 167-74

17 Shepherd NA, Jass JR, Duval I, Moskowitz RL, Nicholls RJ, Morson BC. Restorative proctocolectomy with ileal reservoir: pathological and histochemical study of mucosal biopsy specimens. F Clin Pathol 1987; 40: 601-7.

18 Sandborn WJ, Tremaine WJ, Batts KP, Pemberton JH, Phillips SF. Pouchitis following ileal pouch-anal anastomosis: a pouchitis disease activity index. Mayo Clin Proc 1994; 69: 409-15.

19 Zins BJ, Sandborn WJ, Penna CR, Landers CJ, Targan SR, Tremaine WJ, et al. Pouchitis disease course and antineutrophil cytoplasmic antibody serology following liver transplantation in patients with primary sclerosing 
cholangitis and an ileal pouch-anal anastomosis. Gastroenterology 1995; 108: A949.

20 Sandborn WJ. Pouchitis following ileal pouch-anal anastomosis: definition, pathogenesis, and treatment. Gastroenterology 1994; 107: 1856-60.

21 O'Connell PR, Rankin DR, Weiland LH, Kelly KA. Enteric bacteriology, absorption, morphology and emptying after

22 Boerr LA, Sambuelli A, Filinger E, Peredo H, Kogan Z, Graziano A, et al. Increased mucosal level of leukotriene B4 in pouchitis. Evidences of a persistent chronic inflammatory state. Gastroenterology 1994; 106: A654.

23 Boerr LA, Sambuelli A, Sugai E, Kogan Z, Valero J, Graziano A, et al. Fecal concentration of $\alpha_{1}$-antitrypsin $\left(\left[\alpha_{1}-\mathrm{AT}\right]\right)$ in the diagnosis and management of pouchitis. Gastroenterology 1994; 106: A654

24 Sandborn WJ, Tremaine WJ, Gores GJ, Batts KP, Pemberton JH, Rossi SS, et al. Fecal bile acids, short chain fatty acids, and bacteria after ileal pouch-anal anastomosis do not differ in patients with pouchitis. Dig Dis Sci 1995; 40: 1474-83.

25 Sandborn WJ, Landers CJ, Tremaine WJ, Targan SR. Antineutrophil cytoplasmic antibody correlates with Antineutrophil cytoplasmic antibody correlates with
chronic pouchitis after ileal pouch-anal anastomosis. $A m \mathcal{F}$ Gastroenterol 1995; 90: 740-7.
26 Duerr RH, Targan SR, Landers CJ, LaRusso NF, Lindsay KL, Wiesner RH, et al. Neutrophil cytoplasmic antibodies: a link between primary sclerosing cholangitis and ulcerative colitis. Gastroenterology 1991; 100: 1385-91.

27 Vecchi M, Gionchetti P, Bianchi MB, Belluzzi A, Meucci $\mathrm{G}$, Campieri $\mathrm{M}$, et al. p-ANCA and development of pouchitis in ulcerative colitis patients after proctocolectomy and ileoanal pouch anastomosis. Lancet 1994; 344:

28 Hill MJ, Owen RW. Faecal bile acids in pouch and pouchitis patients: pouchitis workshop. Int $\mathcal{f}$ Colorectal Dis 1989; 4: 221-2.

29 Becker JM, Man CH, Hocking J. Fecal bile acid composition with patients with pouchitis following colectomy and ileal pouch-anal anastomosis. Gastroenterology 1994; 106: A650.

30 Sandborn WJ, Lindor KD, Wiesner RH, LaRusso NF. Ulcerative colitis (UC) disease activity after treatment of associated primary sclerosing cholangitis (PSC) with ursodeoxycholic acid (URSO) or placebo. Gastroenterology 1992; 102: A690.

31 Wiesner RH, LaRusso NF, Dozois RR, Beaver SJ. Peristomal varices after proctocolectomy in patients with primary sclerosing cholangitis. Gastroenterology 1986; 90: primary scie 